severe presentation was described in one of nine infants with haematological signs and symptoms of congenital syphilis. ${ }^{5}$

The negative results of the antenatal serological tests imply that the mother was either incubating the infection at that time, or had acquired it later in pregnancy. A similar sequence of events was seen in five of seven women whose infants were later diagnosed as having early congenital syphilis in a survey from the Mersey region, ${ }^{1}$ which raises the question of whether serological screening should be repeated during the third trimester.

The case we report emphasises the serious implications of early congenital syphilis, and shows that the haematological abnormalities may sometimes be severe.

We thank the department of illustration, Birmingham Children's
Hospital, Jacqueline Boaler for preparation of the manuscript, and Dr JC Clay for helpful discussion.

References

${ }^{1}$ Ewing CI, Roberts C, Davidson DC, Arya OP. Early congenital syphilis still occurs. Arch Dis Child 1985;60:1128-33.

2 World Health Organisation. Treponemal infections. Report of a WHO Scientific Group. Geneva, 1982:674.

${ }^{3}$ Hira SK, Bhat GJ, Patel JB, et al. Early congenital syphilis: clinico radiologic features in 202 patients. Sex Transm Dis 1985;12:177-83.

${ }^{4}$ Stockman JA. Hematologic manifestation of systemic diseases. In: Nathan DG, Oski FA, eds. Hematology of infancy and childhood. Philadelpha: WB Saunders, 1981:1339-87.

5 Whitaker JA, Sartain P, Shaheedy M. Hematological aspects of congenital syphilis. J Pediatr 1965;66:629-36.

Correspondence to Dr MCG Stevens, Department of Oncology, Children's Hospital, Ladywood, Birmingham B16 8ET

Received 26 May 1987

\title{
Post-streptococcal glomerulonephritis in Hong Kong
}

\author{
D T Y LEUNG, R Y M TSENG, S H GO, G L FRENCH, AND C W K LAM
}

Departments of Paediatrics, Microbiology, and Chemical Pathology, Prince of Wales Hospital, The Chinese University of Hong Kong, Shatin, New Territories, and Department of Paediatrics, United Christian Hospital, Kwun Tong, Kowloon

SUMMARY Of 74 paediatric inpatients with acute glomerulonephritis, $58(78 \%)$ had a raised $(>1 / 200)$ antistreptolysin $O$ titre. Serum C3 concentration was low in 73 , but returned to normal within six weeks. Streptococcal infection remains the commonest cause of acute nephritis in children in Hong Kong, possibly due to overcrowded living conditions.

Over the past 20 years the incidence of acute glomerulonephritis in developed countries has fallen considerably, and the aetiological role of streptococcal infection seems also to have waned. Meadow in 1975 found that only. one third of 43 children in Leeds with acute nephritis had had a recent streptococcal infection. ${ }^{1}$

The pattern of illness in children in Hong Kong is similar to that in other developed parts of the world, ${ }^{2}$ yet it was thought that acute glomerulonephritis was more common than in Western countries. We therefore set up a prospective study to determine whether recent streptococcal infection remains a major cause of acute glomerulonephritis in these children.

\section{Patients and methods}

From July 1984 to July 1986 we studied all children with acute glomerulonephritis admitted to two regional hospitals, one in Kowloon and the other in the New Territories. Criteria for diagnosis comprised gross or microscopic haematuria and at least two of the following: diastolic blood pressure of $>80$ $\mathrm{mmHg}$, plasma urea concentration $>6.6 \mathrm{mmol} / \mathrm{l}$, oedema or oliguria. ${ }^{1}$ Children with primary renal disorders were not included.

Seventy four children with suspected acute nephritis were studied: 43 boys and 31 girls, aged from 8 months to 15 years. Particular attention was taken of any throat, respiratory tract, or skin infections in the previous four weeks. We also asked if the children had seen a doctor before admission, and if they had received any medication. Throat swabs for culture and blood specimens for estimation of antistreptolysin O (ASO) titre and assay of C3 complement concentration in serum were taken within five days of admission. Throat swabs were placed in Stuart's transport medium before being sent to the laboratory for culture on blood agar in carbon dioxide. ASO titre was assayed using a modified microtechnique with a microtitre plate; a 
titre of $>1 / 200$ was considered to be diagnostic of streptococcal infection. Serum C3 complement was assayed by rate immunonephelometry using the Beckman ICS-II analyser and reagents. All children except 12 with acute nephritis had at least two samples of blood taken for measurement of ASO titre and $\mathrm{C} 3$ complement concentration within six weeks of admission.

Where possible we enrolled other children as controls. Sixty six control children were studied, having been matched for sex and age (within one year of an index case). These were children whose admission to hospital was within one week of a study case, who were not suffering from a renal complaint, but who required venepuncture as part of the management of their clinical problem. Each had serum ASO titre and C3 complement concentration determined, and a throat swab was taken for culture.

\section{Results}

In 60 patients the chief complaint was swelling of the face, hands, or feet. In only eight was discolouration of the urine the chief symptom. One child presented with a convulsion due to hypertensive encephalopathy. In 36 there was a definite history of a recent throat infection; in 14 skin seemed to be the likely primary focus of infection. Forty nine patients had a diastolic blood pressure of $>80 \mathrm{mmHg}$ on admission. Ninety per cent of the children had consulted a doctor before admission, and over half had been given a broad spectrum antibiotic. All the children recovered in hospital.

ASO titres of $>1 / 200$ were found in $52(70 \%)$ of the study patients on admission compared with 12 $(18 \%)$ of controls. In a further six patients ASO titres that were $<1 / 200$ on admission rose to $>1 / 200$ or more within a month. Serum C3 complement concentrations were low in all but one of the study group on admission, with a mean (SD) value of 24 (15) $\mathrm{mg} / \mathrm{dl}$ (normal range 60-180 mg/dl). Each second specimen of 62 paired blood samples had a C3 concentration higher than the one obtained on admission. Serum C3 complement concentrations gradually returned to normal by the sixth week in all children from whom samples were collected (62), with a mean (SD) value of $89(23) \mathrm{mg} / \mathrm{dl}$.

Group A $\beta$-haemolytic streptococci were isolated from cultures of 10 throat swabs taken from patients with acute nephritis and from six of the controls. We attribute the low recovery rate of organisms from the throat swab cultures to the widespread practice of prescribing broad spectrum antibiotics before admission to hospital. Treatment with antibiotics could also have delayed the rise of the ASO titre, reducing the sensitivity of this method for the diagnosis of preceding streptococcal infection. ${ }^{3}$

\section{Discussion}

Streptococcal infection continues to be the commonest cause of acute glomerulonephritis in children in Hong Kong, as it is in other parts of south east Asia. ${ }^{4}$ Of the 74 patients studied, we were able to prove a recent streptococcal infection (diagnosed by a high ASO titre of $>1 / 200)$ in $58(78 \%)$. A low serum $\mathrm{C} 3$ complement concentration within the first week of presentation is considered by some to be a more reliable test for post-streptococcal glomerulonephritis ${ }^{5}$; all but one of the 74 children fulfilled this criterion.

The importance of the $\beta$-haemolytic streptococcus as a cause of acute glomerulonephritis in our children contrasts with Meadow's experience in Leeds where only one third had post-streptococcal glomerulonephritis. ${ }^{1}$ Environmental conditions in Hong Kong may partly explain these differences. Despite considerable improvement in living conditions the density in terms of number of people per unit area of living space is still much higher than in Western countries, encouraging spread of streptococcal infection. Paradoxically the other major streptococcal disease, rheumatic fever, has virtually disappeared from Hong Kong. This raises the possibility that the high incidence of post-streptococcal glomerulonephritis might also be related to the persistence of nephritogenic strains of $\beta$-haemolytic streptococci in Hong Kong. In the present study we did not determine the types of streptococci, and further studies are in progress to investigate this aspect.

We thank Professor DP Davies and Dr PLS Ip for allowing us to study their patients, and Miss Fanny Lau for preparing the manuscript.

\section{References}

1 Meadow SR. Post-streptococcal nephritis-a rare disease? Arch Dis Child 1975;50:379-81.

2 Leung DTY, Tseng RYM, Davies DP. Setting up a clinical audit of paediatric morbidity in Hong Kong: some early experiences. Aust Paediatr J 1987;23:111-3.

${ }^{3}$ McCarty M. The antibody response to streptococcal infections. Streptococcal infection. New York: Columbia Press, 1954.

4 Tanphaichitr P, Chatasingh S. Post-streptococcal nephritis-still not a rare disease in Thailand. Arch Dis Child 1976;51:484-5.

5 Cameron JS, Vick RM, Ogg CS, Seymour WM, Chantler C, Turner DR. Plasma C3 and C4 concentrations in management of glomerulonephritis. Br Med J 1973;3:668-72.

Correspondence to Dr DTY Leung, Department of Paediatrics, The Chinese University of Hong Kong, Prince of Wales Hospital, Shatin, New Territories, Hong Kong.

Received 12 May 1987 